\title{
AN ASYMPTOTIC PHASE FOR A STURM-LIOUVILLE OPERATOR WITH UNBOUNDED POTENTIAL
}

\author{
RICHARD C. GILBERT
}

1. Introduction. If $\phi\left(x, s^{2}\right)$ is the solution of $L y=-y^{\prime \prime}-q(x) y$ $=s^{2} y, y(0)=0, y^{\prime}(0)=-1$, where the potential $q$ is defined on $[0, \infty)$ and decreases in magnitude sufficiently rapidly as $x$ tends to infinity, then $\phi\left(x, s^{2}\right)=C(s) \sin (s x-\alpha(s))+o(1)$ as $x$ tends to infinity (see [5, Lemma 5]). The function $\alpha$ is called the asymptotic phase. Given the asymptotic phase, the potential $q$ can be reconstructed. Buslaev and Faddeev [2] and Gilbert and Kramer [5] proved that the asymptotic phase satisfies the equation

$$
\sum_{j=1}^{n}-k_{j}^{2}+(2 / \pi) \int_{0}^{\infty}\left[s \alpha(s)-(1 / 2) \int_{0}^{\infty} q(x) d x\right] d s=-q(0) / 4,
$$

where the $-k_{j}^{2}$ are the eigenvalues of the operator in $L^{2}(0, \infty)$ defined by $L$ and the boundary condition $y(0)=0$.

Equation (1) is known as a "trace" formula. Similar formulas have been obtained by many other authors in different situations; e.g., by Buslaev [1] for a three-dimensional Schroedinger operator with a potential decreasing in magnitude sufficiently rapidly at infinity, and by Gasymov [4] and Gilbert and Kramer [6] for operators with a discrete spectrum.

In this article we show that it is possible to define an asymptotic phase for the solution $\phi(x, \lambda)$ of $-y^{\prime \prime}+[-x+p(x)] y=\lambda y, y(0)=0$, $y^{\prime}(0)=-1$, where $p$ is a finite ${ }^{1}$ function on $[0, \infty)$. The asymptotic phase is absolutely integrable and satisfies an equation similar to (1). As is indicated by Gasymov [3], perturbation by a finite function of the operator $-y^{\prime \prime}-x^{t} y, 0<t \leqq 2$, produces other interesting phenomena.

We note that our formula (17) for the trace of the difference of the resolvents in terms of the asymptotic phase is the analog of formula (11) of Kreǐn [7]. Kreǐn's formula was deduced for abstract operators and involves his "spectral shift" function. Formulas similar to (17) which involve an asymptotic phase have been obtained by Buslaev and Faddeev [2] and by Buslaev [1].

Presented to the Society, August 9, 1965 ; received by the editors October 13, 1965.

${ }^{1}$ By a finite function is meant a function which vanishes outside some finite interval. 
2. The basic solutions. Let $f_{1}(\lambda)=(\pi \lambda / 3)^{1 / 2} J_{1 / 3}(Z)$ and $f_{2}(\lambda)$ $=(\pi \lambda / 3)^{1 / 2} Y_{1 / 3}(Z)$, where $Z=(2 / 3) \lambda^{3 / 2}$ and $J_{1 / 3}$ and $Y_{1 / 3}$ are Bessel functions. $f_{1}(x+\lambda)$ and $f_{2}(x+\lambda)$ are linearly independent solutions of $-y^{\prime \prime}-x y=\lambda y$. If $\phi_{1}(z, \lambda)$ is the solution of $L_{1} y=-y^{\prime \prime}-x y=\lambda y$, $y(0)=0, y^{\prime}(0)=-1$, then

$$
\phi_{1}(x, \lambda)=f_{2}(\lambda) f_{1}(x+\lambda)-f_{1}(\lambda) f_{2}(x+\lambda) .
$$

We observe that $f_{1}$ and $f_{2}$ are entire functions of $\lambda$, real for real $\lambda$, and never simultaneously zero. Their Wronskian is 1 . If $\lambda$ is real, then as $\lambda \rightarrow+\infty$

$$
\begin{aligned}
f_{1}(\lambda) & =\lambda^{-1 / 4}[\cos (Z-5 \pi / 12)+O(1 / Z)], \\
f_{1}^{\prime}(\lambda) & =\lambda^{1 / 4}[-\sin (Z-5 \pi / 12)+O(1 / Z)], \\
f_{2}(\lambda) & =\lambda^{-1 / 4}[\sin (Z-5 \pi / 12)+O(1 / Z)], \\
f_{2}^{\prime}(\lambda) & =\lambda^{1 / 4}[\cos (Z-5 \pi / 12)+O(1 / Z)] .
\end{aligned}
$$

If $Z^{\prime}=(2 / 3) \mu^{3 / 2}$, then as $\mu \rightarrow+\infty$

$$
\begin{aligned}
f_{1}(-\mu) & =(1 / 2) \mu^{-1 / 4}\left[-1+O\left(1 / Z^{\prime}\right)\right] \exp Z^{\prime}, \\
f_{1}^{\prime}(-\mu) & =(1 / 2) \mu^{1 / 4}\left[1+O\left(1 / Z^{\prime}\right)\right] \exp Z^{\prime}, \\
f_{2}(-\mu) & =\left(3^{1 / 2} / 2\right) \mu^{-1 / 4}\left[-1+O\left(1 / Z^{\prime}\right)\right] \exp Z^{\prime}, \\
f_{2}^{\prime}(-\mu) & =\left(3^{1 / 2} / 2\right) \mu^{1 / 4}\left[1+O\left(1 / Z^{\prime}\right)\right] \exp Z^{\prime} .
\end{aligned}
$$

Let $p$ be a real continuous function on $[0, \infty)$ which vanishes for $x \geqq c$. If $\phi_{2}(x, \lambda)$ is the solution of $L_{2} y=-y^{\prime \prime}+[-x+p(x)] y=\lambda y$, $y(0)=0, y^{\prime}(0)=-1$, then for $x \geqq c$,

$$
\phi_{2}(x, \lambda)=A(\lambda) f_{1}(x+\lambda)-B(\lambda) f_{2}(x+\lambda) .
$$

We have that

$$
\begin{aligned}
& A(\lambda)=\phi_{2}(c, \lambda) f_{2}^{\prime}(c+\lambda)-\phi_{2}{ }^{\prime}(c, \lambda) f_{2}(c+\lambda), \\
& B(\lambda)=\phi_{2}(c, \lambda) f_{1}^{\prime}(c+\lambda)-\phi_{2}^{\prime}(c, \lambda) f_{1}(c+\lambda) .
\end{aligned}
$$

We see that $A(\lambda)$ and $B(\lambda)$ are entire in $\lambda$, real for real $\lambda$, never simultaneously zero.

Let $q$ be a continuous function on $[0, c]$. Let $\phi(x, \lambda)$ be the solution of $-y^{\prime \prime}+q(x) y=\lambda y, y(0)=0, y^{\prime}(0)=-1$. According to Titchmarsh [8, Section 1.7] as $s \rightarrow+\infty, \phi\left(x, s^{2}\right)=-(1 / s)$ sin $s x+O\left(1 / s^{2}\right)$, $\phi^{\prime}\left(x, s^{2}\right)=-\cos s x+O(1 / s)$. As $t \rightarrow+\infty$,

$$
\begin{aligned}
\phi\left(x,-t^{2}\right) & =(1 / 2 t) e^{t x}\left[-1+e^{-2 t x}+O(1 / t)\right], \\
\phi^{\prime}\left(x,-t^{2}\right) & =(1 / 2) e^{t x}\left[-1-e^{-2 t x}+O(1 / t)\right] .
\end{aligned}
$$


If $q$ is continuously differentiable, we can say, further, that $\phi\left(x, s^{2}\right)$ $=-(1 / s) \sin s x+\left(1 / 2 S^{2}\right) \int_{0}^{x} q(y) d y \cos s x+O\left(1 / s^{3}\right)$. Each result holds uniformly for $x$ in $[0, c]$.

Using (4) and (5) and the asymptotics we have given for $f_{1}, f_{2}$ and $\phi$, we see that as $\lambda \rightarrow+\infty, A(\lambda)=\lambda^{-1 / 4}\left[\sin (Z-5 \pi / 12)+O\left(\lambda^{-1 / 2}\right)\right]$, $B(\lambda)=\lambda^{-1 / 4}\left[\cos (Z-5 \pi / 12)+O\left(\lambda^{-1 / 2}\right)\right]$. As $\mu \rightarrow+\infty$, we see that

$$
\begin{aligned}
& A(-\mu)=\left(3^{1 / 2} / 2\right) \mu^{-1 / 4}\left[-1+O\left(\mu^{-1 / 2}\right)\right] \exp Z^{\prime}, \\
& B(-\mu)=(1 / 2) \mu^{-1 / 4}\left[-1+O\left(\mu^{-1 / 2}\right)\right] \exp Z^{\prime} .
\end{aligned}
$$

3. The basic operators and their spectral functions. Let $T_{j}$ be the self-adjoint operator in $L^{2}(0, \infty)$ generated by the differential operator $L_{j}$ and the boundary condition $y(0)=0$. Following the methods of Titchmarsh [8, Section 4.13] we see that if $\rho_{j}(\lambda)$ is the spectral function of $T_{j}$ as defined in [2], then for all real $\lambda$,

$$
\begin{aligned}
& \rho_{1}^{\prime}(\lambda)=(1 / \pi)\left[f_{1}^{2}(\lambda)+f_{2}^{2}(\lambda)\right]^{-1}, \\
& \rho_{2}^{\prime}(\lambda)=(1 / \pi)\left[A^{2}(\lambda)+B^{2}(\lambda)\right]^{-1} .
\end{aligned}
$$

As $\lambda \rightarrow+\infty$,

$\rho_{1}^{\prime}(\lambda)=(1 / \pi) \lambda^{1 / 2}[1+O(1 / Z)]$ and $\rho_{2}^{\prime}(\lambda)=(1 / \pi) \lambda^{1 / 2}\left[1+O\left(\lambda^{-1 / 2}\right)\right]$. As $\mu \rightarrow+\infty$,

$$
\begin{aligned}
& \rho_{1}^{\prime}(-\mu)=(1 / \pi) \mu^{1 / 2}\left[1+O\left(1 / Z^{\prime}\right)\right] \exp \left(-4 \mu^{3 / 2} / 3\right), \\
& \rho_{2}^{\prime}(-\mu)=(1 / \pi) \mu^{1 / 2}\left[1+O\left(\mu^{-1 / 2}\right)\right] \exp \left(-4 \mu^{3 / 2} / 3\right) .
\end{aligned}
$$

4. The asymptotic phase. We can define two real analytic functions $\alpha_{1}(\lambda)$ and $\alpha_{2}(\lambda),-\infty<\lambda<\infty$, by means of the equations $\tan \alpha_{1}(\lambda)$ $=f_{2}(\lambda) / f_{1}(\lambda)$, tan $\alpha_{2}(\lambda)=A(\lambda) / B(\lambda)$. Letting

$$
\psi_{j}(x, \lambda)=\left[\rho_{j}^{\prime}(\lambda)\right]^{1 / 2} \phi_{j}(x, \lambda),
$$

we see that for $x \geqq c$,

(8) $\psi_{j}(x, \lambda)=\left(1 / \pi^{1 / 2}\right)\left[f_{1}(x+\lambda) \sin \alpha_{j}(\lambda)-f_{2}(x+\lambda) \cos \alpha_{j}(\lambda)\right]$,

where $j=1,2$.

We have that $\tan \left(\alpha_{2}-\alpha_{1}\right)=h_{2} / h_{1}$, where $h_{2}(\lambda)=A(\lambda) f_{1}(\lambda)$ $-B(\lambda) f_{2}(\boldsymbol{\lambda})$ and $h_{1}(\boldsymbol{\lambda})=B(\boldsymbol{\lambda}) f_{1}(\boldsymbol{\lambda})+A(\boldsymbol{\lambda}) f_{2}(\boldsymbol{\lambda})$. Since

$$
\left(\begin{array}{ll}
\phi_{2}(c, \lambda) & \phi_{1}(c, \lambda) \\
\phi_{2}^{\prime}(c, \lambda) & \phi_{1}^{\prime}(c, \lambda)
\end{array}\right)=\left(\begin{array}{ll}
f_{1}(c+\lambda) & f_{2}(c+\lambda) \\
f_{1}^{\prime}(c+\lambda) & f_{2}^{\prime}(c+\lambda)
\end{array}\right)\left(\begin{array}{rr}
A(\lambda) & f_{2}(\lambda) \\
-B(\lambda) & -f_{1}(\lambda)
\end{array}\right),
$$

we see that 


$$
\begin{aligned}
h_{2}(\lambda) & =\phi_{1}(c, \lambda) \phi_{2}^{\prime}(c, \lambda)-\phi_{1}^{\prime}(c, \lambda) \phi_{2}(c, \lambda) \\
& =\int_{0}^{c} p(x) \phi_{2}(x, \lambda) \phi_{1}(x, \lambda) d x .
\end{aligned}
$$

Assume now that $p \in C^{2}[0, c]$ and that $\int_{0}^{c} p(x) d x=0$. From our asymptotics for $\phi_{1}$ and $\phi_{2}$ it follows that $h_{2}(\lambda)=O\left(1 / \lambda^{2}\right)$ as $\lambda \rightarrow+\infty$, and $h_{2}(-\mu)=O\left[\mu^{-1} \exp \left(2 c \mu^{1 / 2}\right)\right]$ as $\mu \rightarrow+\infty$. From our asymptotics for $A, B, f_{1}, f_{2}$, we see that $h_{1}(\lambda)=\lambda^{-1 / 2}\left[1+O\left(\lambda^{-1 / 2}\right)\right]$ as $\lambda \rightarrow+\infty$, and $h_{1}(-\mu)=\left[1+O\left(\mu^{-1 / 2}\right)\right] \mu^{-1 / 2} \quad \exp \left(4 \mu^{3 / 2} / 3\right)$ as $\mu \rightarrow+\infty$. Hence $\tan \left[\alpha_{2}(\lambda)-\alpha_{1}(\lambda)\right]=O\left(\lambda^{-3 / 2}\right)$ as $\lambda \rightarrow+\infty$, and $\tan \left[\alpha_{2}(-\mu)-\alpha_{1}(-\mu)\right]$ $=O\left[\mu^{-1 / 2} \exp \left(2 c \mu^{1 / 2}-(4 / 3) \mu^{3 / 2}\right)\right]$ as $\mu \rightarrow+\infty$. Therefore, there are integers $n_{1}, n_{2}$ such that $\alpha_{2}(\lambda)-\alpha_{1}(\lambda) \rightarrow n_{1} \pi$ as $\lambda \rightarrow+\infty$, and $\alpha_{2}(-\mu)$ $-\alpha_{1}(-\mu) \rightarrow n_{2} \pi$ as $\mu \rightarrow+\infty$. Let $\delta(\lambda)=\alpha_{2}(\lambda)-\alpha_{1}(\lambda)-n_{1} \pi$ for $\lambda>0$, and let $\delta(\lambda)=\alpha_{2}(\lambda)-\alpha_{1}(\lambda)-n_{2} \pi$ for $\lambda<0$. We call $\delta(\lambda)$ the asymptotic phase. We have that $\delta(\lambda)=O\left(1 / \lambda^{3 / 2}\right)$ as $\lambda \rightarrow+\infty$, and

$$
\delta(-\mu)=O\left[\mu^{-1 / 2} \exp \left(2 c \mu^{1 / 2}-(4 / 3) \mu^{3 / 2}\right)\right]
$$

as $\mu \rightarrow+\infty$. We note $\delta(\lambda)$ is absolutely integrable from $-\infty$ to $+\infty$.

5. The trace of the difference of the resolvents. Let $R_{j}(z)$ be the resolvent of $T_{j} . R_{j}(z)$ is an integral operator with kernel $K_{j}(x, y, z)$ $=\int_{-\infty}^{\infty}(\lambda-z)^{-1} \phi_{j}(x, \lambda) \phi_{j}(y, \lambda) d \rho_{j}(\lambda)$. Let $V$ be the operator in $L^{2}(0, \infty)$ of multiplication by $p$. If \|\|$_{2}$ denotes the Schmidt norm, then $\left\||V|^{1 / 2} R_{1}(i \tau)\right\|_{2}^{2}=\int_{0}^{c}|p(x)| \int_{-\infty}^{\infty}|\lambda-i \tau|^{-2} \phi_{1}^{2}(x, \lambda) \rho_{1}^{\prime}(\lambda) d \lambda d x$. We may express the inner integral as the sum of three integrals, taken from $-\infty$ to $-b$, from $-b$ to $b$, and from $b$ to $+\infty$. Using our asymptotics for $\phi_{1}(x, \lambda)$ and $\rho_{1}^{\prime}(\lambda)$, we can then show that $\left\||V|^{1 / 2} R_{1}(i \tau)\right\|_{2}$ $=O\left(\tau^{\epsilon-3 / 4}\right)$, where $\epsilon$ is an arbitrarily small positive number. By Gilbert and Kramer [5, Theorem 1], it follows that $R_{2}(i \tau)-R_{1}(i \tau)$ is in the trace class, and, in addition, that

$$
\lim _{\tau \rightarrow \infty} \tau^{2}\left[S\left\{R_{2}(i \tau)-R_{1}(i \tau)\right\}+S\left\{R_{1}(i \tau) V R_{1}(i \tau)\right\}\right]=0,
$$

where $S$ stands for trace.

We have that

$$
\begin{aligned}
S\left\{R_{2}(i \tau)-R_{1}(i \tau)\right\} & =\int_{0}^{\infty}\left[K_{2}(x, x, i \tau)-K_{1}(x, x, i \tau)\right] d x \\
& =\int_{0}^{\infty} \int_{-\infty}^{\infty}(\lambda-i \tau)^{-1}\left[\psi_{2}^{2}(x, \lambda)-\psi_{1}^{2}(x, \lambda)\right] d \lambda d x .
\end{aligned}
$$

Since the integrals 


$$
\int_{-\infty}^{\infty}(\lambda-i \tau)^{-1} \psi_{j}^{2}(x, \lambda) d \lambda, \quad j=1,2,
$$

converge uniformly for $0 \leqq x \leqq N$, where $N$ is an arbitrary positive number, it follows that

$$
\begin{aligned}
S\left\{R_{2}(i \tau)-R_{1}(i \tau)\right\}= & \lim _{N \rightarrow \infty} \int_{-\infty}^{\infty}(\lambda-i \tau)^{-1} \\
& \cdot\left[\int_{0}^{N} \psi_{2}^{2}(x, \lambda) d x-\int_{0}^{N} \psi_{1}^{2}(x, \lambda) d x\right] d \lambda .
\end{aligned}
$$

As in Gilbert and Kramer [5], $\int_{0}^{N} \psi_{j}^{2}(x, \lambda) d x=\psi_{j, 1}(N, \lambda) \psi_{j, 2}(N, \lambda)$ $-\psi_{j}(N, \lambda) \psi_{j, 12}(N, \lambda)$, where the subscripts after the ' $j$ ' stand for partial derivatives. Assuming that $N \geqq c$, calculating the partial derivatives from (8) and substituting into (10), we obtain

$$
\begin{aligned}
S\left\{R_{2}(i \tau)-R_{1}(i \tau)\right\}= & (1 / \pi) \lim _{N \rightarrow \infty} \int_{-\infty}^{\infty}(\lambda-i \tau)^{-1} \\
& \cdot\left\{(N+\lambda)\left[a_{2}^{2}(N, \lambda)-a_{1}^{2}(N, \lambda)\right]\right. \\
+ & \left.b_{2}^{2}(N, \lambda)-b_{1}^{2}(N, \lambda)-\left[\alpha_{2}^{\prime}(\lambda)-\alpha_{1}^{\prime}(\lambda)\right]\right\} d \lambda,
\end{aligned}
$$

where $a_{j}(N, \lambda)=f_{1}(N+\lambda) \sin \alpha_{j}(\lambda)-f_{2}(N+\lambda) \cos \alpha_{j}(\lambda)$, and $b_{j}(N, \lambda)$ $=f_{1}^{\prime}(N+\lambda) \sin \alpha_{j}(\lambda)-f_{2}^{\prime}(N+\lambda) \cos \alpha_{j}(\lambda)$. Integration by parts shows that

$$
\begin{aligned}
\int_{-\infty}^{\infty}(\lambda-i \tau)^{-1}\left[\alpha_{2}^{\prime}(\lambda)\right. & \left.-\alpha_{1}^{\prime}(\lambda)\right] d \lambda \\
= & {\left[\delta\left(0^{+}\right)-\delta\left(0^{-}\right)\right] / i \tau+\int_{-\infty}^{\infty}(\lambda-i \tau)^{-2} \delta(\lambda) d \lambda . }
\end{aligned}
$$

Making the change of variable $u=\lambda+N$ and letting $k$ be an arbitrary positive number, we can write

$$
\begin{aligned}
\int_{-\infty}^{\infty}(\lambda-i \tau)^{-1}\{(N & \left.+\lambda)\left[a_{2}^{2}(N, \lambda)-a_{1}^{2}(N, \lambda)\right]+b_{2}^{2}(N, \lambda)-b_{1}^{2}(N, \lambda)\right\} d \lambda \\
& =\int_{-\infty}^{\infty}(u-N-i \tau)^{-1} g(u, N) d u \\
& =\left[\int_{-\infty}^{-k}+\int_{-k}^{k}+\int_{k}^{\infty}\right](u-N-i \tau)^{-1} g(u, N) d u,
\end{aligned}
$$


where $g(u, \quad N)=u\left[a_{2}^{2}(N, \quad u-N)-a_{1}^{2}(N, u-N)\right]+b_{2}^{2}(N, \quad u-N)$ $-b_{1}^{2}(N, u-N)$. It is then clear that

$$
\lim _{N \rightarrow \infty} \int_{-k}^{k}(u-N-i \tau)^{-1} g(u, . v) d u=0 .
$$

Using our asymptotics for $f_{1}, f_{2}$ and their derivatives, we see that $g(u, N)=O(1 / u)$ as $u \rightarrow+\infty$, uniformly in $N$. Assuming that $k$ was chosen large enough, we therefore have $\left|\int_{\mathbf{k}}^{\infty}(u-N-i \tau)^{-1} g(u, N) d u\right|$ $\leqq M \int_{\mathfrak{k}}^{\infty}\left[(u-N)^{2}+\tau^{2}\right]^{-1 / 2} u^{-1} d u$. This last integral may be expressed in terms of elementary functions. It is then possible to show that its limit is zero as $N \rightarrow \infty$. Hence, $\lim _{N \rightarrow \infty} \int_{k}^{\infty}(u-N-i \tau)^{-1} g(u, N) d u=0$. Using our asymptotics for $f_{1}, f_{2}, f_{1}^{\prime}, f_{2}^{\prime}, \delta$, we see that $g(-v, N)$ $=O\left(1 / v^{3 / 2}\right)$ as $v \rightarrow+\infty$, uniformly in $N$ for $N$ sufficiently large. Therefore,

$$
\begin{aligned}
\lim _{N \rightarrow \infty} \int_{-\infty}^{-k} & (u-N-i \tau)^{-1} g(u, N) d u \\
& =\lim _{N \rightarrow \infty} \int_{k}^{\infty}(-v-. V-i \tau)^{-1} g\left(-\tau^{\prime}, . Y\right) d r=0 .
\end{aligned}
$$

We have thus shown that

$$
\begin{aligned}
\lim _{N \rightarrow \infty} \int_{-\infty}^{\infty}(\lambda-i \tau)^{-1}\left\{(X+\lambda)\left[a_{2}^{2}(N, \lambda)-a_{1}^{2}(N, \lambda)\right]\right. \\
\left.+b_{2}^{2}(N, \lambda)-b_{1}^{2}(Y, \lambda)\right\} d \lambda=0 .
\end{aligned}
$$

From (11), (12) and (13) it follows that

$$
\begin{aligned}
S\left\{R_{2}(i \tau)-R_{1}(i \tau)\right\}= & {\left[\delta\left(0^{-}\right)-\delta\left(0^{+}\right)\right] / i \pi \tau } \\
& -(1 / \pi) \int_{-\infty}^{\infty}(\lambda-i \tau)^{-2} \delta(\lambda) d \lambda .
\end{aligned}
$$

6. A trace formula. Using the asymptotics we have given for $\phi_{1}$ and $\rho_{1}^{\prime}$, we observe that $\int_{0}^{c} p(x) \phi_{1}^{2}(x, \lambda) d x \rho_{1}^{\prime}(\lambda)$ is $O\left(1 / \lambda^{3 / 2}\right)$ as $\lambda \rightarrow+\infty$, and if $\lambda=-\mu$, it is $O\left(\mu^{-1 / 2} \exp \left[2 c \mu^{1 / 2}-(4 / 3) \mu^{3 / 2}\right]\right)$ as $\mu \rightarrow+\infty$. Hence, $\int_{0}^{c} p(x) \phi_{1}^{2}(x, \lambda) d x \rho_{1}^{\prime}(\lambda)$ is absolutely integrable for $-\infty<\lambda<\infty$, and by Gilbert and Kramer [5, Theorem 2] we have that

$$
\lim _{\tau \rightarrow \infty} \tau^{2} S\left\{R_{1}(i \tau) V R_{1}(i \tau)\right\}=-\int_{-\infty}^{\infty} \int_{0}^{c} p(x) \phi_{1}^{2}(x, \lambda) d x d \rho_{1}(\lambda) .
$$

From equations (9), (14) and (15) it follows that $\delta\left(0^{+}\right)=\delta\left(0^{-}\right)$ and that 


$$
(1 / \pi) \int_{-\infty}^{\infty} \delta(\lambda) d \lambda=-\int_{-\infty}^{\infty} \int_{0}^{c} p(x) \phi_{1}^{2}(x, \lambda) d x d \rho_{1}(\lambda) .
$$

We may summarize our results in the following form:

Theorem. Let $R_{j}(z), j=1,2$, be the resolvent and $\rho_{j}$ the spectral function of the operator in $L^{2}(0, \infty)$ defined by the differential operator $L_{j} y=-y^{\prime \prime}+[-x+(j-1) p(x)] y$ and the boundary condition $y(0)=0$, where $p$ is real, $p \in C^{2}[0, c], p(x)=0$ for $x \geqq c, \int_{0}^{c} p(x) d x=0$. If $\phi_{j}(x, \lambda)$ is the solution of $L_{j} y=\lambda y, y(0)=0, y^{\prime}(0)=-1$, then for $x \geqq c$,

$$
\phi_{j}(x, \lambda)=[\pi(x+\lambda) / 3]^{1 / 2}\left[A_{j}(\lambda) J_{1 / 3}(X)-B_{j}(\lambda) Y_{1 / 3}(X)\right],
$$

where $Y=(2 / 3)(x+\lambda)^{3 / 2}$. Let $\alpha_{j}(\lambda)$ be a real analytic function defined by the equations $\tan \alpha_{j}(\lambda)=A_{j}(\lambda) / B_{j}(\lambda), \lim _{\lambda \rightarrow \pm \infty}\left[\alpha_{2}(\lambda)-\alpha_{1}(\lambda)\right]=0$. Then $\delta(\lambda)=\alpha_{2}(\lambda)-\alpha_{1}(\lambda)$ is called the asymptotic phase. $\delta(\lambda)$ is absolutely integrable, and the following equations are true:

$$
S\left\{R_{2}(i \tau)-R_{1}(i \tau)\right\}=-(1 / \pi) \int_{-\infty}^{\infty}(\lambda-i \tau)^{-2} \delta(\lambda) d \lambda,
$$

where $S$ stands for trace, and

$$
(1 / \pi) \int_{-\infty}^{\infty} \delta(\lambda) d \lambda=-\int_{-\infty}^{\infty} \int_{0}^{c} p(x) \phi_{1}^{2}(x, \lambda) d x d \rho_{1}(\lambda) .
$$

\section{REFERENCES}

1. V. S. Buslaev, Trace formulas for Schroedinger's operator in three-dimensional space, Dokl. Akad. Nauk SSSR 143 (1962), 1067-1070.

2. V. S. Buslaev and L. D. Faddeev, Formulas for traces for a singular SturmLiouville operator, Dokl. Akad. Nauk SSSR 132 (1960), 13-16.

3. M. G. Gasymov, On the analytic properties of the spectral function of a selfadjoint Sturm-Liouville operator, Dokl. Akad. Nauk SSSR 150 (1963), 971-974= Soviet Math. Dokl. 4 (1963), 780-783.

4. - On the sum of the differences of the eigenvalues of two self-adjoint operators, Dokl. Akad. Nauk SSSR 150 (1963), 1202-1205=Soviet Math. Dokl. 4 (1963), $838-842$.

5. R. C. Gilbert and V. A. Kramer, Trace formulas for a perturbed operator, Duke Math. J. 30 (1963), 275-296.

6. - Trace formulas for powers of a Sturm-Liouville operator, Canad. J. Math. 16 (1964), 412-422.

7. M. G. KreY̌n, On perturbation determinants and trace formulas for unitary and self-adjoint operators, Dokl. Akad. Nauk SSSR 144 (1962), 268-271=Soviet Math. Dokl. 3 (1962), 707-710.

8. E. C. Titchmarsh, Eigenfunction expansions, Oxford Univ. Press, New York, 1946.

California State College at Fullerton 